Check for updates

Cite this: RSC Adv., 2019, 9, 5302

\title{
First-principles calculations of mechanical and thermodynamic properties of tetragonal $\mathrm{Be}_{12} \mathrm{Ti}$
}

\author{
Xiankun Liu, (D) *a Qijie Feng, ${ }^{a}$ Bin Tang, ${ }^{a}$ Jian Zheng, ${ }^{a} Z^{2 h o u ~ Z h e n g, ~}{ }^{b}$ Wei Zhou, ${ }^{a}$ \\ Jiting Tian $^{a}$ and Jing Wang ${ }^{c}$
}

The elastic and thermodynamic properties of tetragonal $\mathrm{Be}_{12} \mathrm{Ti}$ under high temperature and pressure are investigated by first-principles calculations based on pseudopotential plane-wave density functional theory (DFT) within the generalized gradient approximation (GGA) and quasi-harmonic approximation (QHA). The calculated lattice parameters and bulk modulus are in good agreement with the available experimental data. The calculated elastic constants of $\mathrm{Be}_{12} \mathrm{Ti}$ increase monotonously with increasing pressure, and the elastic stability criterion and the phonon dispersion calculation show that the $\mathrm{Be}_{12} \mathrm{Ti}$ crystal satisfies the mechanical and dynamic stability under applied pressure (0-100 GPa). The related mechanical properties such as bulk modulus $(B)$, shear modulus $(G)$, Young's modulus $(E)$, and Poisson's ratio $(\nu)$ are also studied for polycrystalline of $\mathrm{Be}_{12} \mathrm{Ti}$; the calculated $B / G$ value shows that $\mathrm{Be}_{12} \mathrm{Ti}_{\text {behaves }}$ in a brittle manner, and higher pressure can significantly improve the brittleness of $\mathrm{Be}_{12} \mathrm{Ti}$. The elastic anisotropy is demonstrated by the elastic anisotropy factors. The direction-dependent Young's modulus and bulk modulus of $\mathrm{Be}_{12} \mathrm{Ti}$ are dealt with in detail under pressure from $0 \mathrm{GPa}$ to $100 \mathrm{GPa}$. The pressure and temperature dependencies of the relative volume, the bulk modulus, the elastic constants, the heat capacity and the thermal expansion coefficient, as well as the entropy are obtained and discussed using the quasi-harmonic approximation in the ranges of temperature $0-1600 \mathrm{~K}$ and pressure $0-100 \mathrm{GPa}$.

Received 21st October 2018
Accepted 4th February 2019

DOI: $10.1039 / \mathrm{c} 8 \mathrm{ra08711c}$

rsc.li/rsc-advances properties of the materials, ${ }^{10,11}$ it can be produced phase transitions and changes of physical and chemical properties in the materials. ${ }^{12,13}$ From early literature, more research has focused on experimental aspects for $\mathrm{Be}_{12} \mathrm{Ti}$, especially for the fabrication and tritium release under normal conditions. ${ }^{14-17}$ Although there are a few theoretical calculations for Be-Ti alloys, they mainly focus on hexagonal $\mathrm{Be}_{12}$ Ti alloy, ${ }^{18,19}$ as for the tetragonal $\mathrm{Be}_{12} \mathrm{Ti}$, its elastic and thermodynamic properties under high temperature and pressure are missing. Hence, it is necessary to obtain the elastic anisotropy and thermodynamic properties of tetragonal $\mathrm{Be}_{12} \mathrm{Ti}$ alloy at high temperature and high pressure. The first-principles density functional theory method (DFT) is a useful tool for predicting the physical properties of the solid materials, particularly elastic and thermodynamic properties, and it has been widely applied in various materials. ${ }^{20-27}$

In this present work, the effects of temperature and pressure on the structure, elasticity and thermodynamic properties of tetragonal $\mathrm{Be}_{12} \mathrm{Ti}$ alloys are studied by using the first-principles method and quasi-harmonic approximation (QHA). The calculated results are expected to provide valuable estimation for the experimental and theoretical research of $\mathrm{Be}_{12} \mathrm{Ti}$ intermetallic compound. The rest paper is organized as follows: in Section 2, we have given the theoretical method and the computation details. Some results and discussions are described in Section 3. Finally, the summary of our main results and conclusions is summarized in Section 4.

\footnotetext{
${ }^{a}$ Institute of Nuclear Physics and Chemistry, CAEP, Mianyang 621900, China. E-mail xiankunliu@126.com; xiankunliu@caep.cn

${ }^{b}$ Institute of Chemical Materials, CAEP, Mianyang 621900, China

'Institute of Materials, CAEP, Mianyang 621900, China
} 


\section{Computational methods and details}

In present work, all calculations are performed by firstprinciples based on VASP software package, ${ }^{28}$ which is based on density functional theory (DFT). We describe the interaction between core ions and valence electrons using PAW pseudopotentials ${ }^{29,30}$ and the exchange-correlation function are treated by the generalized gradient approximation (GGA) within Perdew-Burke-Ernzerhof (PBE). The plane wave cut-off energy was taken as $550 \mathrm{eV}$. Pseudo atomic calculations of $\mathrm{Be}_{12} \mathrm{Ti}$ are performed for Be $2 s^{2}$ and $\mathrm{Ti} 3 \mathrm{~s}^{2} 3 \mathrm{p}^{6} 3 \mathrm{~d}^{2} 4 \mathrm{~s}^{2}$. For the Brillouin-zone sampling, we use the $8 \times 8 \times 14$ Monkhorst-Pack mesh for $k$ space integration, where the self-consistent convergence of the total energy is set to $1.0 \times 10^{-6} \mathrm{eV}$ per atom, the maximum

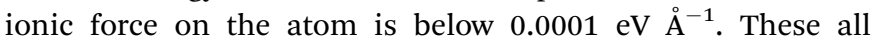
parameters including $k$-point convergence test have been carefully tested.

In order to obtain the equilibrium volume and bulk modulus of, the $E-V$ results obtained from the first calculation will be fitted by the following Birch-Murnaghan EOS: ${ }^{31}$

$$
\begin{aligned}
E(V)= & E_{0}-\frac{9}{16} B_{0}\left[\left(4-B_{0}^{\prime}\right) \frac{V_{0}^{3}}{V^{2}}-\left(14-3 B_{0}^{\prime}\right) \frac{V_{0}^{7 / 3}}{V^{4 / 3}}\right. \\
& \left.+\left(16-3 B_{0}^{\prime}\right) \frac{V_{0}^{5 / 3}}{V^{2 / 3}}\right]
\end{aligned}
$$

where $E_{0}$ is the equilibrium energy at $P=0$, the $B_{0}$ is the bulk modulus, $B_{0}^{\prime}$ is the pressure derivative on bulk modulus. As for elastic properties, here we have used stress-strain approach based on the Hooke's law to calculate the elasticity, in which the elastic stiffness tensor $\left[C_{i j}\right]$ can be expressed as ${ }^{32}$

$$
\left\{\delta_{i j}\right\}=\left[C_{i j}\right]\left\{\varepsilon_{i j}\right\}
$$

where $\left\{\delta_{i j}\right\}$ and $\left\{\varepsilon_{i j}\right\}$ are stress and strain tensors respectively. For tetragonal $\mathrm{Be}_{12} \mathrm{Ti}$ crystal, there are six independent components $\left(C_{11}, C_{12}, C_{13}, C_{33}, C_{44}\right.$ and $\left.C_{66}\right)$. Based on above $C_{i j}$ values, other elastic parameters for example bulk modulus $B$ and shear modulus $G$ and Young's modulus $E$ can be obtained by Voigt-
Reuss-Hill approximation from elastic constants in ref. 33 and 34.

\section{Results and discussion}

\subsection{Crystal structure and stability}

The crystal structure of $\mathrm{Be}_{12} \mathrm{Ti}$ in this study belongs to the tetragonal with space group $I 4 / \mathrm{mmm}^{35}$ in its unit cell, the Be atoms occupy Wyckoff position $8 \mathrm{f}(0.25,0.25,0.25), 8 \mathrm{i}(0.35,0$, $0), 8 \mathrm{j}(0.28,0.5,0)$ and $\mathrm{Ti}$ atoms occupy $2 \mathrm{a}(0,0,0)$, respectively (see Fig. 1). In order to determine the equilibrium structure of $\mathrm{Be}_{12} \mathrm{Ti}$, we first carried out the structural optimization calculation under different pressure (pressure range of 0-100 GPa), obtained the corresponding energy $E$ and volume $V$. Then, fitted above the $E$ and $V$ to the Birch-Murnaghan equation of state $(\text { EOS })^{29}$ to get the bulk modulus $\left(B_{0}\right)$ and its pressure derivative $\left(B_{0}^{\prime}\right)$ at $0 \mathrm{~K}$ and $0 \mathrm{GPa}$. Fitted energy $E$ - volume $V$ relations of $\mathrm{Be}_{12} \mathrm{Ti}$ are shown in Fig. 2, and the fitting results of the bulk $B_{0}$ and its derivative $B_{0}^{\prime}$ and equilibrium lattice constants are given in Table 1. It can be seen from Table 1 that the calculated lattice constants $\left(a\right.$ and $c$ ) and bulk modulus $\left(B_{0}\right)$ are very close to the previous experimental data ${ }^{35,36}$ even the experimental values were obtained in the $273 \mathrm{~K}$ environment, while pressure derivative $\left(B_{0}^{\prime}\right)$ has not yet been compared with the experimental data. Nevertheless, this work is a significant attempt to predict the characteristics of tetragonal $\mathrm{Be}_{12} \mathrm{Ti}$ alloys. It can provide reference data for further research on the properties of $\mathrm{Be}_{12} \mathrm{Ti}$ alloy.

Usually, the pressure can deform the material and even cause the phase transition of the material. But for different materials, the effect of pressure on the structure parameters is different. For tetragonal $\mathrm{Be}_{12} \mathrm{Ti}$, there are differences in lattice constants and volume changes $\left(a / a_{0}, c / c_{0}, V / V_{0}\right)$ under external pressure. Fig. 3 shows the variation of $a / a_{0}, c / c_{0}$, and $V / V_{0}$ with pressure in the range of 0 to $100 \mathrm{GPa}$, where $a_{0}, c_{0}$ and $V_{0}$ denote the equilibrium structural parameters and cell volume at $T=$ $0 \mathrm{~K}$ and $P=0 \mathrm{GPa}$, respectively. It can be seen that with increasing pressure, the equilibrium ratios $a / a_{0}, c / c_{0}$ and $V / V_{0}$ decrease smoothly, but $V / V_{0}$ decrease quickly than $c / c_{0}$ and $a / a_{0}$, and $c / c_{0}$ decreases slightly faster than $a / a_{0}$, indicating that the

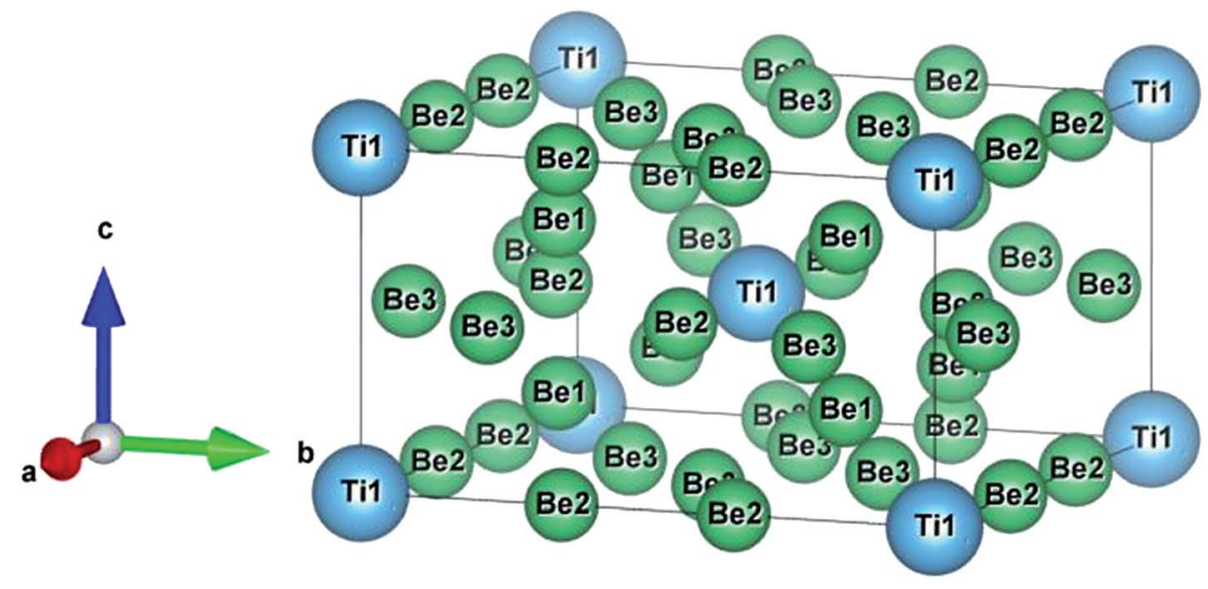

Fig. 1 Crystal structure of the tetragonal $\mathrm{Be}_{12} \mathrm{Ti}$. 


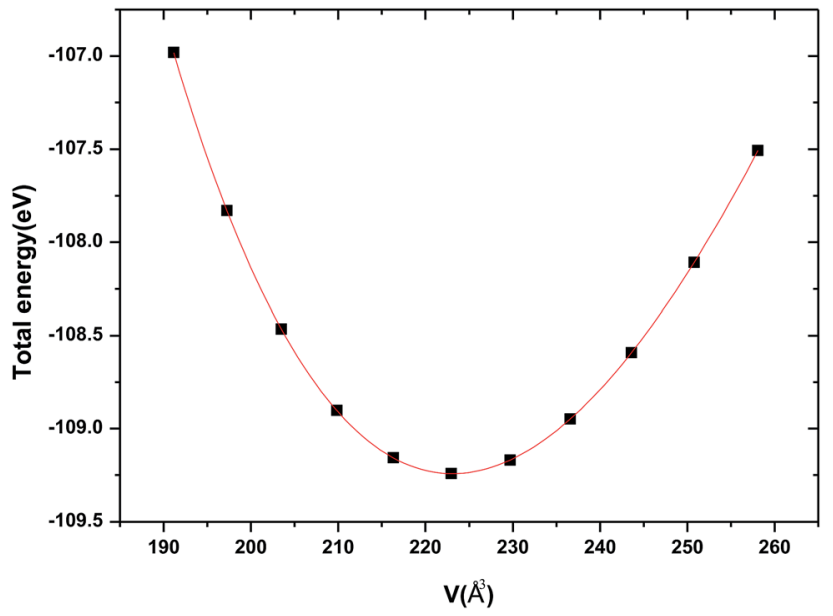

Fig. 2 The calculated total energy $E$ varies unit cell volume $V$ for tetragonal $\mathrm{Be}_{12} \mathrm{Ti}$.

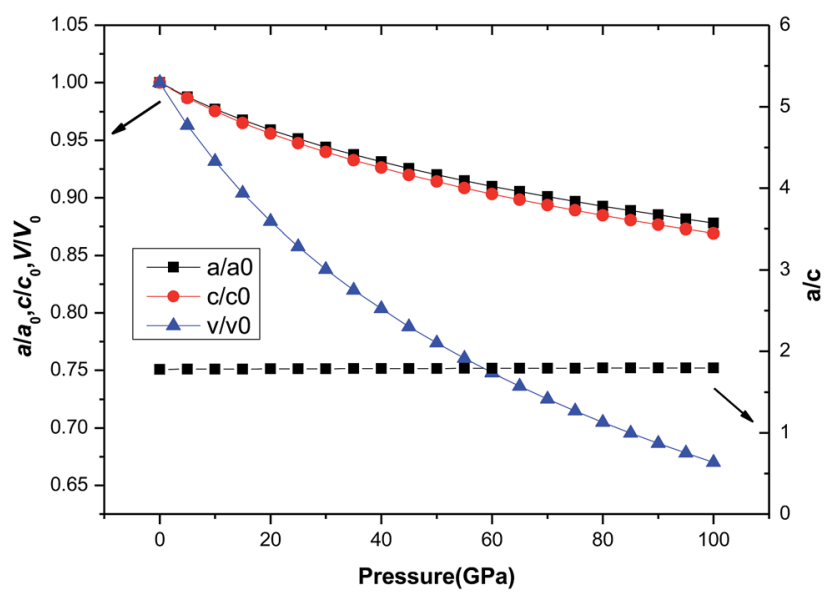

Fig. 3 The dependence of normalized lattice constants $a / a_{0}, c / c_{0}$, and cell volume $V / V_{0}$ on pressure for tetragonal $\mathrm{Be}_{12} \mathrm{Ti}$.

compression along the $a$-axis is slightly smaller than that along the $c$-axis. This suggests that the lattice constant $c$ is more sensitive to external pressure than $a$. Nevertheless, change value of the $a / c$ is not large and it remains almost constant in the range of pressure from 0 to $100 \mathrm{GPa}$.

\subsection{Elastic properties}

The elastic property is important parameter which is related to many physical properties of the solid materials, including specific heat, Debye temperature, thermal expansion, Grüneisen parameter and melting point. Some mechanical properties and dynamical behaviors of the materials can be obtained by its elastic constants. For tetragonal $\mathrm{Be}_{12} \mathrm{Ti}$, there are six independent elastic constants $C_{11}, C_{12}, C_{13}, C_{33}, C_{44}$ and $C_{66}$. In the Fig. 4(a) and (b), we presented the elastic constants and its increasing rate with pressure of $\mathrm{Be}_{12} \mathrm{Ti}$ under various pressures. From Fig. 4(a), we can see that all elastic constants increase with applied pressure up to $100 \mathrm{GPa}$ for $\mathrm{Be}_{12} \mathrm{Ti}$. The increasing rate of each elastic constant with pressure is derived from the slope of the corresponding linear fitted curve, as shown in Fig. 4(b). Among them, the variation of $C_{11}$ is the most sensitive at 4.62 , while $C_{13}$ has the least increasing rate at 0.67 . According to calculated elastic constants and mentioned formulas in ref. 34, we have calculated the mechanical properties of $\mathrm{Be}_{12} \mathrm{Ti}$ under various pressure, such as bulk modulus $B$, shear modulus $G$, Young's modulus $E$, and Poisson's ration $\nu$, which are listed with other theoretical data in Table 2. It is obvious from Table 2 that the Voigt-Reuss-Hill approximations bulk modulus $B_{\mathrm{H}}(114.27 \mathrm{GPa})$ at $0 \mathrm{GPa}$ is very close to the value $B_{0}$ $(121.0 \mathrm{GPa})$ obtained with above Birch-Murnaghan EOS $^{\mathbf{3 1}}$ and experimental data. ${ }^{36}$ It indicates that our calculations are reliable and calculation method is correct. On the other hand, the bulk modulus $B$ and shear modulus $G$ can represent the resistance to volume change and shape change, respectively. The Young's modulus is related to the stiffness of materials. From Table 2, we also found that the bulk modulus $B$ and shear modulus $G$ and Young's modulus $E$ increase with growing pressures till $40 \mathrm{GPa}$, indicating that the increase of resistance ability to volume and shape, and the stiffness of $\mathrm{Be}_{12} \mathrm{Ti}$ is getting harder and harder, and the $\mathrm{Be}_{12} \mathrm{Ti}$ becomes more and more difficult to compress as pressure increases. At the same time the value of $B$ is greater than $G$, implies the principal parameter limiting the stability of tetragonal $\mathrm{Be}_{12} \mathrm{Ti}$ is the shear modulus. ${ }^{37}$

For a tetragonal crystal, the traditional mechanical stability conditions under isotropic pressure are given by the following equations: ${ }^{38}$

$\tilde{C}_{11}>0, \tilde{C}_{33}>0, \tilde{C}_{44}>0, \tilde{C}_{66}>0, \tilde{C}_{11}-\tilde{C}_{12}>0,\left(\tilde{C}_{33}+\tilde{C}_{11}-\right.$ $\left.2 \tilde{C}_{13}\right)>0,\left(2 \tilde{C}_{11}+2 \tilde{C}_{12}+\tilde{C}_{33}+4 \tilde{C}_{13}\right)>0$

where $\tilde{C}_{\alpha \alpha}=C_{\alpha \alpha}-P(a=1,3,4,6), \tilde{C}_{12}=C_{12}+P, \tilde{C}_{13}=C_{13}+P$. It is evident that from calculated results the elastic constants of $\mathrm{Be}_{12} \mathrm{Ti}$ satisfy all of these conditions above at pressure up to $100 \mathrm{GPa}$, which indicates that tetragonal structure $\mathrm{Be}_{12} \mathrm{Ti}$ under applies pressure is elastic stable. In order to determine the

Table 1 Calculated equilibrium lattice constants a and $c(\AA)$, equilibrium volume $V_{0}\left(\AA^{3}\right)$, bulk modules $B_{0}(G P a)$ and its pressure derivative $B_{0}^{\prime}$ (GPa) of $\mathrm{Be}_{12} \mathrm{Ti}$ at zero pressure

\begin{tabular}{llllll}
\hline Phase & & $a(\AA)$ & $c(\AA)$ & $V_{0}\left(\AA^{3}\right)$ & $B_{0}(\mathrm{GPa})$ \\
\hline $\mathrm{Be}_{12} \mathrm{Ti}$ & This calc. & 7.25 & 4.17 & 223.097 & 121.0 \\
& Experimental $(T=273 \mathrm{~K})$. & $7.35^{a}$ & $4.19^{a}$ & 226.354 & $117^{b}$
\end{tabular}

${ }^{a}$ Ref. $35 .{ }^{b}$ Ref. 36. 

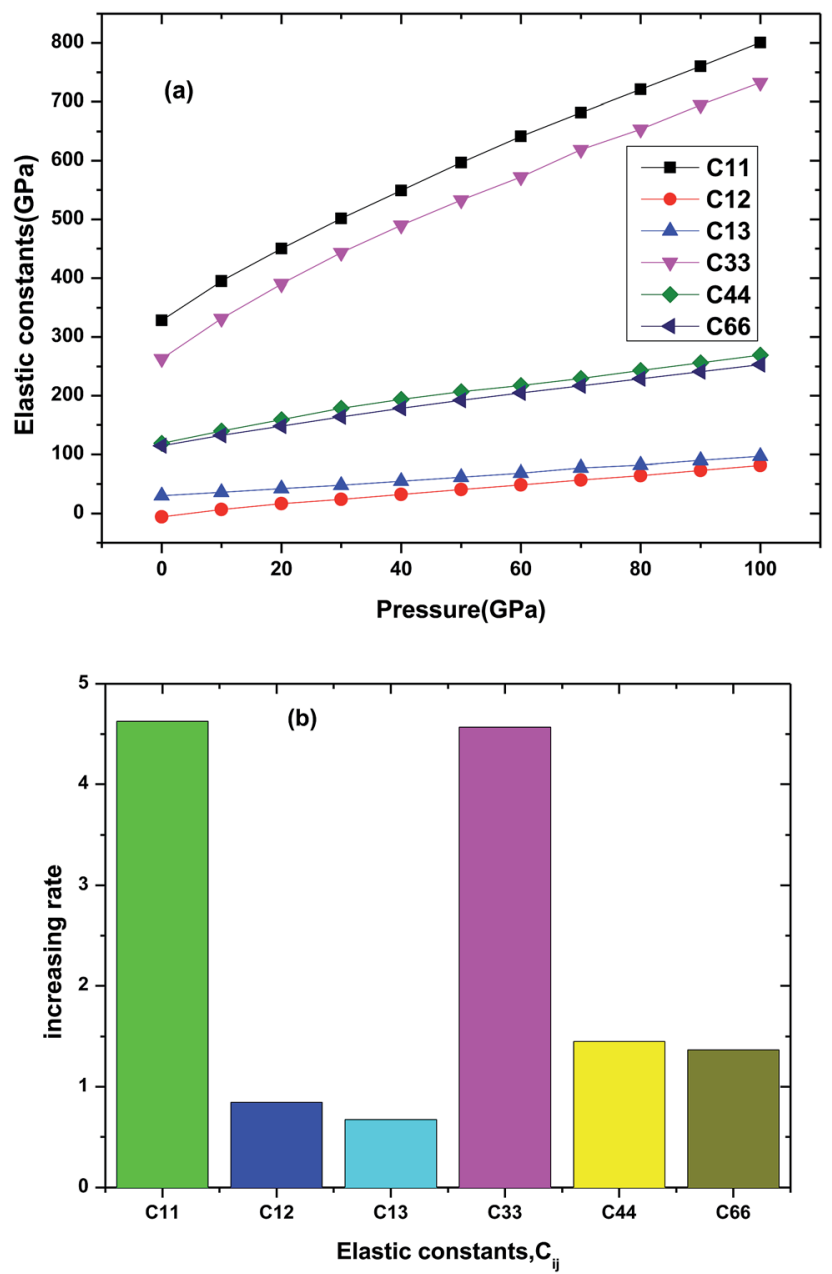

Fig. 4 The dependencies of elastic constants (a) and increasing rate (b) for each elastic constant for $\mathrm{Be}_{12} \mathrm{Ti}$.

dynamic stability under the pressure considered, we also calculate the phonon dispersion based on linear response (DFPT) for the $2 \times 2 \times 2$ supercell with 104 atoms at 0, 20, 40,
$60 \mathrm{GPa}$ and $100 \mathrm{GP}$ in Fig. 5 where no imaginary frequencies in the whole Brillouin zone of $\mathrm{Be}_{12} \mathrm{Ti}$ and do not show any anomaly for all the phonon modes, confirming that $\mathrm{Be}_{12} \mathrm{Ti}$ with tetragonal structure is at least stable dynamically up to $100 \mathrm{GPa}$. Meanwhile, we found that the acoustic and optical modes become more compact and frequency range increase as the pressure increasing, when the pressure reaches $100 \mathrm{GPa}$, the phonon spectrum intervals between acoustic and optical modes have disappeared.

Poisson's ratio $\nu$ can be used to estimate the ductility and brittleness of the materials. As for $\mathrm{Be}_{12} \mathrm{Ti}$, its Poisson's ratio value has been listed in Table 2, we can see that from Table 2 all Poisson's ratio values are less than $1 / 3$ under applied pressure, which means that the $\mathrm{Be}_{12} \mathrm{Ti}$ is brittle in nature. ${ }^{39}$ Meanwhile, according to the Pugh's criterion, ${ }^{41}$ the ratio of the bulk modulus to the shear modules $B / G$ can be used to predict the whether a material will behave in a brittle or ductile manner. A high $B / G$ ratio is associated with ductility, whereas a low value corresponds to a brittle material, the critical value between them is around $1.75{ }^{40}$ If $B / G$ is less than 1.75 , then brittle behavior is predicted; otherwise, the material behaves in a ductile manner. In this study, calculated $B / G$ values for tetragonal $\mathrm{Be}_{12} \mathrm{Ti}$ as a function of pressure are shown in Table 2 and Fig. 6. It is noted that the value of $B / G$ is 0.90 at $0 \mathrm{~K}$ and $0 \mathrm{GPa}$, showing that tetragonal $\mathrm{Be}_{12} \mathrm{Ti}$ has a brittle nature under these conditions. When applied pressure increases from $0 \mathrm{GPa}$ to $100 \mathrm{GPa}, B / G$ monotonously increases from 0.90 to 1.09 , which is always remaining smaller than 1.75 . This trend is markedly different from ref. 17 . This indicates that $\mathrm{Be}_{12} \mathrm{Ti}$ will also behave in a brittle manner at pressure up to $100 \mathrm{GPa}$, but it has a tendency towards the development of ductility, indicating that the external pressure can improve the brittleness of tetragonal $\mathrm{Be}_{12} \mathrm{Ti}$ alloys.

\subsection{Elastic anisotropy}

Anisotropic behavior has an important implication in engineering science as well as crystal physics since it is highly related with the possibility to induce microcracks in

Table 2 The calculated elastic constants $C_{i j}(\mathrm{GPa})$, bulk modulus $B(\mathrm{GPa})$, shear modulus $G(\mathrm{GPa})$, Young's modulus $E$ (GPa), Zener anisotropy factor $A$ and Poisson's ratio $\nu$ of $\mathrm{Be}_{12} \mathrm{Ti}$ at different pressure

\begin{tabular}{|c|c|c|c|c|c|c|c|c|c|}
\hline$P$ & $B_{\mathrm{V}}$ & $G_{\mathrm{V}}$ & $B_{\mathrm{R}}$ & $G_{\mathrm{R}}$ & $B_{\mathrm{H}}$ & $G_{\mathrm{H}}$ & $E$ & $B / G$ & $\nu$ \\
\hline 0 & 114.40 & 128.14 & 114.13 & 125.61 & $\begin{array}{l}114.27 \\
117.00^{a} \\
121.0^{b}\end{array}$ & 126.87 & 277.80 & 0.90 & 0.09 \\
\hline 10 & 142.07 & 151.86 & 141.80 & 149.02 & 141.93 & 150.44 & 333.50 & 0.94 & 0.11 \\
\hline 20 & 165.75 & 172.42 & 165.51 & 169.30 & 165.63 & 170.86 & 381.43 & 0.97 & 0.12 \\
\hline 30 & 187.36 & 192.43 & 187.15 & 189.09 & 187.25 & 190.76 & 427.21 & 0.98 & 0.12 \\
\hline 40 & 208.09 & 209.57 & 207.86 & 205.95 & 207.97 & 207.76 & 467.57 & 1.00 & 0.13 \\
\hline 50 & 228.34 & 225.41 & 228.06 & 221.37 & 228.20 & 223.39 & 505.29 & 1.02 & 0.13 \\
\hline 60 & 247.49 & 239.05 & 247.15 & 234.49 & 247.32 & 236.77 & 538.47 & 1.04 & 0.14 \\
\hline 70 & 267.28 & 253.14 & 267.04 & 248.28 & 267.16 & 250.71 & 572.92 & 1.07 & 0.14 \\
\hline 80 & 283.56 & 267.42 & 283.25 & 262.45 & 283.41 & 264.94 & 605.98 & 1.07 & 0.14 \\
\hline 90 & 302.27 & 281.59 & 301.99 & 276.49 & 302.13 & 279.04 & 640.08 & 1.08 & 0.15 \\
\hline 100 & 320.45 & 295.48 & 320.12 & 290.17 & 320.29 & 292.83 & 673.29 & 1.09 & 0.15 \\
\hline
\end{tabular}

${ }^{a}$ Ref. 36. ${ }^{b}$ Present work-fitted by BMEOS. 

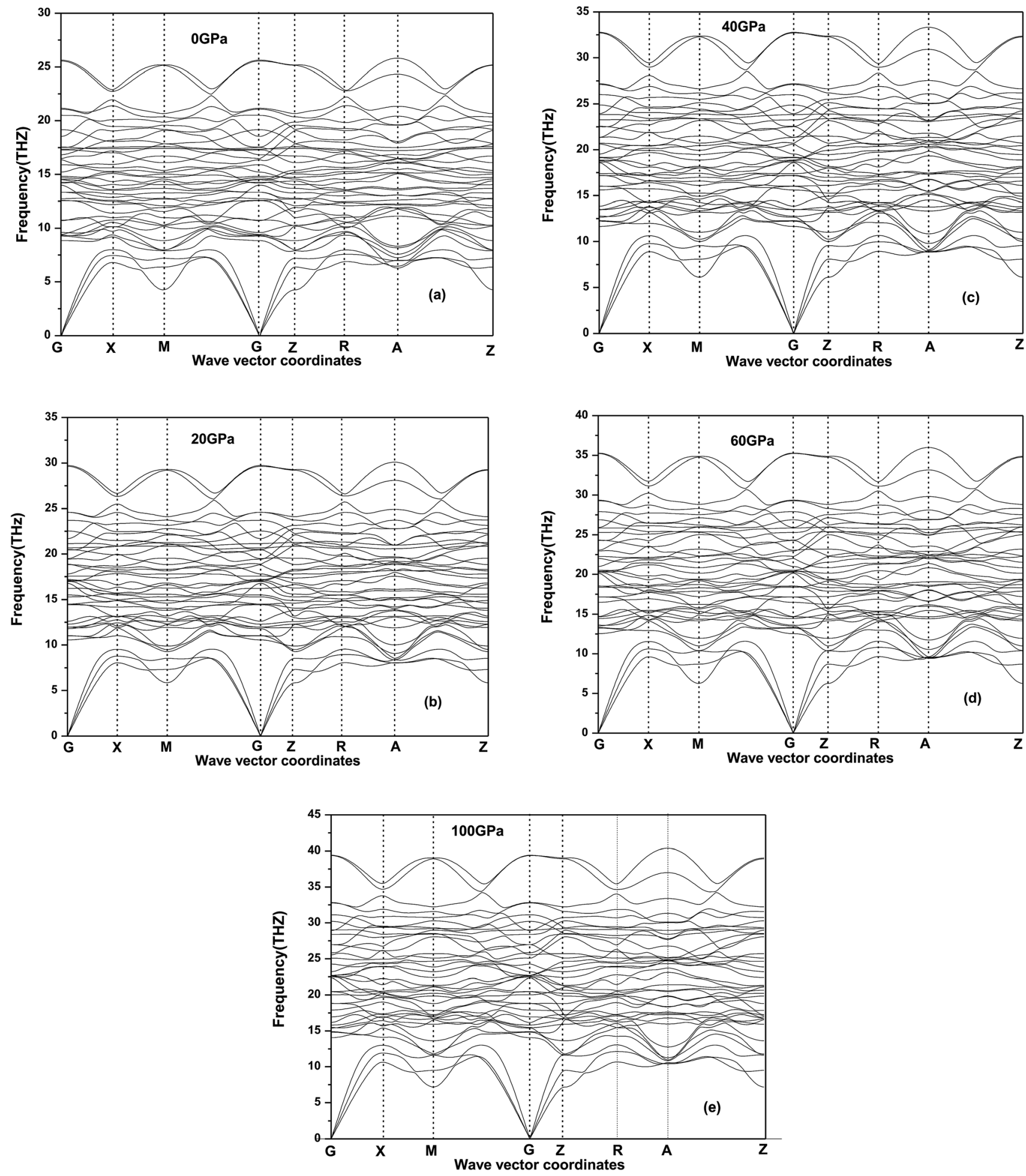

Fig. 5 Phonon dispersion curves for tetragonal $\mathrm{Be}_{12} \mathrm{Ti}$ at $0 \mathrm{GPa}$ (a), $20 \mathrm{GPa}$ (b), $40 \mathrm{GPa}$ (c), $60 \mathrm{GPa}$ (d) and $100 \mathrm{GPa}$ (e).

materials. By means of elastic anisotropy, we can know more information about the material. Such as phase transformation, precipitation, dislocation dynamics, and microcracks formation i.e. ${ }^{41}$ Usually, the elastic anisotropy can be represented by three elastic factors, which are shear anisotropic factors $\left(A_{1}, A_{2}\right.$ and $\left.A_{3}\right)$ of different crystallographic planes and universal anisotropic index $\left(A^{\mathrm{U}}\right)$ and the percentage of anisotropy in compression and shear $\left(A_{\mathrm{B}}\right.$ and $A_{\mathrm{G}}$ ). In this work, we have calculated above three type anisotropy of tetragonal $\mathrm{Be}_{12} \mathrm{Ti}$ under applied pressure and listed in Table 3 , the detail calculation equations as follows ${ }^{42,43}$ 


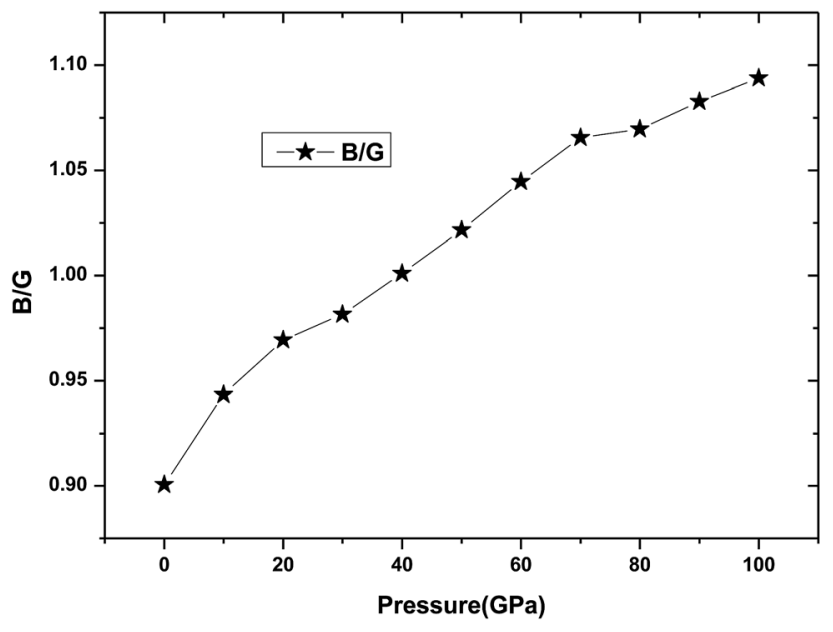

Fig. 6 The dependencies of $B / G$ ratio on pressure for $\mathrm{Be}_{12} \mathrm{Ti}$.

$$
\begin{aligned}
A_{1} & =\frac{4 C_{66}}{C_{11}+C_{22}-2 C_{12}}, A_{2}=\frac{4 C_{55}}{C_{22}+C_{33}-2 C_{23}}, A_{3} \\
& =\frac{4 C_{44}}{C_{11}+C_{33}-2 C_{13}}
\end{aligned}
$$

For a tetragonal structure,

$$
\begin{aligned}
A_{1} & =\frac{2 C_{66}}{C_{11}-C_{12}}, A_{2}=A_{3}=\frac{4 C_{44}}{C_{11}+C_{33}-2 C_{13}} \\
A^{\mathrm{U}} & =5 \frac{G_{\mathrm{V}}}{G_{\mathrm{R}}}+\frac{B_{\mathrm{V}}}{B_{\mathrm{R}}}-6, A_{\mathrm{B}}=\frac{B_{\mathrm{V}}-B_{\mathrm{R}}}{B_{\mathrm{V}}+B_{\mathrm{R}}} \times 100 \%, A_{\mathrm{G}} \\
& =\frac{G_{\mathrm{V}}-G_{\mathrm{R}}}{G_{\mathrm{V}}+G_{\mathrm{R}}} \times 100 \%
\end{aligned}
$$

where the $A_{1}, A_{2}, A_{3}$ are shear anisotropic factor for the (001) and $(010)$ and (100) shear planes, respectively. The $B_{\mathrm{V}}, B_{\mathrm{R}}, G_{\mathrm{V}}$, and $G_{\mathrm{R}}$ stand for the Voigt and Reuss approximation for bulk modulus $(B)$ and shear modulus $(G)$, respectively. For isotropic structural materials, the factors $A_{1}, A_{2}$ and $A_{3}$ should be equated to one, the value of the $A^{\mathrm{U}}$ is also zero, while any deviations from one or zero means the degree of elastic anisotropy of the crystal. The maximum value of $A_{\mathrm{B}}$ and $A_{\mathrm{G}}$ is $100 \%$ and the minimum is zero, the zero value means isotropy, while $100 \%$ represent maximum anisotropy. From Table 3, one can see clearly that $A_{1}$ and $A_{2}$ are smaller than one, when the applied pressure increases, the $A_{1}$ and $A_{2}$ only have slightly changed than that value at zero pressure. For $A^{\mathrm{U}}$, it is seen that $\mathrm{Be}_{12} \mathrm{Ti}$ have the small degree of universal elastic anisotropic behavior, which means $\mathrm{Be}_{12} \mathrm{Ti}$ is slightly anisotropic, and that characteristics are enhanced gradually with the increasing pressure. As for percentage of anisotropy $A_{\mathrm{B}}$ and $A_{\mathrm{G}}$, it is found that as the pressure increases, the anisotropy of $A_{\mathrm{B}}$ almost keep constant, whereas the shear modulus anisotropy $A_{\mathrm{G}}$ increases gently indicating that tetragonal $\mathrm{Be}_{12} \mathrm{Ti}$ is more anisotropy in shear than in compressibility. Meanwhile, the calculated value of $A_{\mathrm{G}}$ is larger that $A_{\mathrm{B}}$ under different pressure, also implying that the shear modulus shows stronger directional dependence than bulk modulus.

Actually, the anisotropy of material also can be revealed by three dimensional curved surfaces of Young's and Bulk modulus on crystallographic directions. For a tetragonal structure $\mathrm{Be}_{12} \mathrm{Ti}$, the direction dependent of Young's modulus $(E)$ and Bulk modulus $(B)$ is defined as follows:

$$
\begin{gathered}
\frac{1}{B}=\left(S_{11}+S_{12}+S_{13}\right)\left(l_{1}{ }^{2}+l_{2}{ }^{2}\right)+\left(2 S_{13}+S_{33}\right) l_{3}{ }^{2} \\
\frac{1}{E}=S_{11}\left(l_{1}{ }^{4}+l_{2}{ }^{4}\right)+\left(2 S_{13}+S_{44}\right)\left(l_{1}{ }^{2} l_{3}{ }^{2}+l_{2}{ }^{2} l_{3}{ }^{2}\right)+S_{33} l_{3}{ }^{4}+\left(2 S_{12}\right. \\
\left.+S_{66}\right) l_{1}{ }^{2} l_{2}{ }^{2}
\end{gathered}
$$

where $S_{i j}$ are the elastic compliance constants, and $l_{1}, l_{2}$ and $l_{3}$ are the directional cosines to the $X, Y$ and $Z$ axes under spherical coordinates, respectively. ${ }^{44}$ The three-dimensional surface of the Young's and Bulk modulus for $\mathrm{Be}_{12} \mathrm{Ti}$ with tetragonal structure at $0 \mathrm{GPa}$ and $40 \mathrm{GPa}$ is shown in Fig. 7 and 8 , respectively. From Fig. 7 and 8, we can see that the distances between the origin of coordinate and the 3D surface are not same in any direction, and the Young's and Bulk modulus have small changes under high pressure, implies that as increasing of pressure from $0 \mathrm{GPa}$ to $40 \mathrm{GPa}$, the elastic anisotropy of

\begin{tabular}{|c|c|c|c|c|c|}
\hline Pressure (GPa) & $A_{1}$ & $A_{2}$ & $A^{\mathrm{U}}$ & $A_{\mathrm{B}}$ & $A_{\mathrm{G}}$ \\
\hline 0 & $6.844 \times 10^{-1}$ & $8.955 \times 10^{-1}$ & $1.031 \times 10^{-1}$ & $1.146 \times 10^{-3}$ & $9.979 \times 10^{-3}$ \\
\hline 10 & $6.825 \times 10^{-1}$ & $8.539 \times 10^{-1}$ & $9.741 \times 10^{-2}$ & $9.800 \times 10^{-4}$ & $9.454 \times 10^{-3}$ \\
\hline 30 & $6.860 \times 10^{-1}$ & $8.406 \times 10^{-1}$ & $8.953 \times 10^{-2}$ & $5.580 \times 10^{-4}$ & $8.764 \times 10^{-3}$ \\
\hline 40 & $6.900 \times 10^{-1}$ & $8.334 \times 10^{-1}$ & $8.907 \times 10^{-2}$ & $5.500 \times 10^{-4}$ & $8.720 \times 10^{-3}$ \\
\hline 50 & $6.902 \times 10^{-1}$ & $8.231 \times 10^{-1}$ & $9.242 \times 10^{-2}$ & $6.080 \times 10^{-4}$ & $9.038 \times 10^{-3}$ \\
\hline 80 & $6.978 \times 10^{-1}$ & $8.027 \times 10^{-1}$ & $9.580 \times 10^{-2}$ & $5.420 \times 10^{-4}$ & $9.383 \times 10^{-3}$ \\
\hline 90 & $7.014 \times 10^{-1}$ & $8.038 \times 10^{-1}$ & $9.313 \times 10^{-2}$ & $4.640 \times 10^{-4}$ & $9.136 \times 10^{-3}$ \\
\hline 100 & $7.035 \times 10^{-1}$ & $8.030 \times 10^{-1}$ & $9.258 \times 10^{-2}$ & $5.030 \times 10^{-4}$ & $9.074 \times 10^{-3}$ \\
\hline
\end{tabular}
$\mathrm{Be}_{12} \mathrm{Ti}$ becomes not more obvious. In addition, we have presented the two-dimensional projections of Young's modulus and bulk modulus on different crystallography planes at $0 \mathrm{GPa}$ and $40 \mathrm{GPa}$ in Fig. 7 and 8, which are consistent with the above conclusions in three-dimensional space.

Table 3 The shear anisotropy factors $\left(A_{1}, A_{2}\right)$, universal elastic anisotropy index $\left(A^{\cup}\right)$ and the percentage of anisotropy $\left(A_{\mathrm{B}}\right.$ and $\left.A_{\mathrm{G}}\right)$ of $\mathrm{Be} \mathrm{e}_{12} \mathrm{Ti}$ under various pressure 


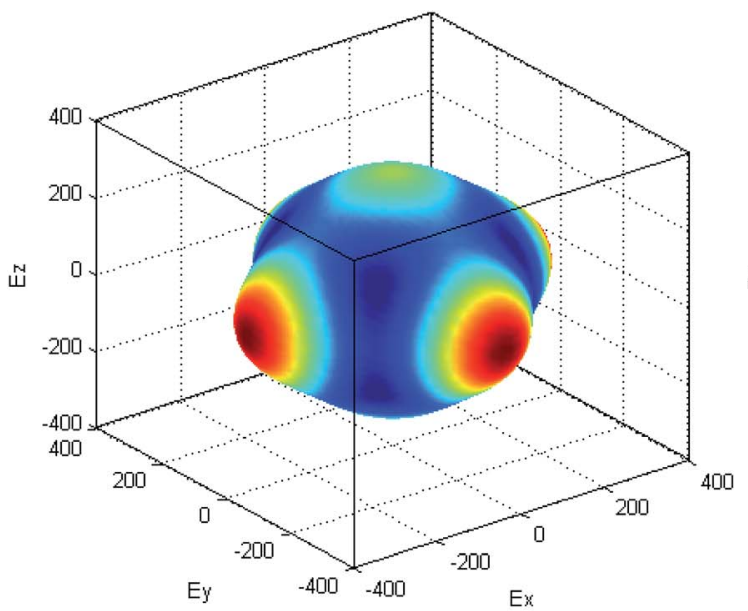

(a)

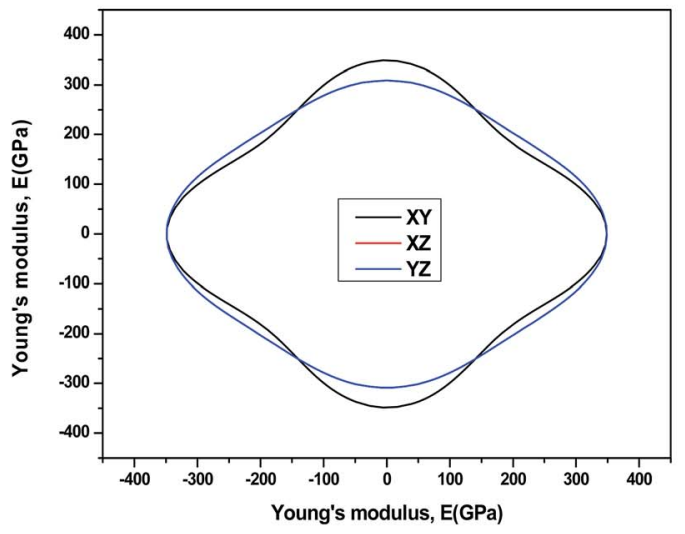

(c)

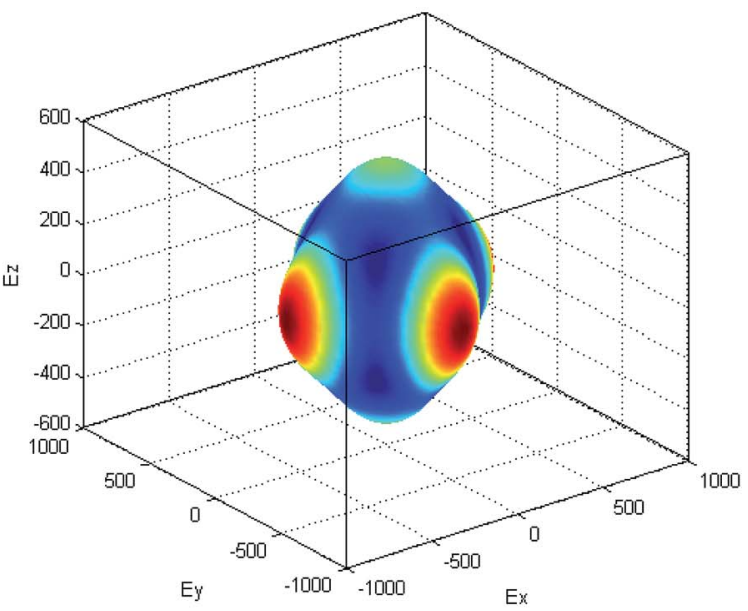

(b)

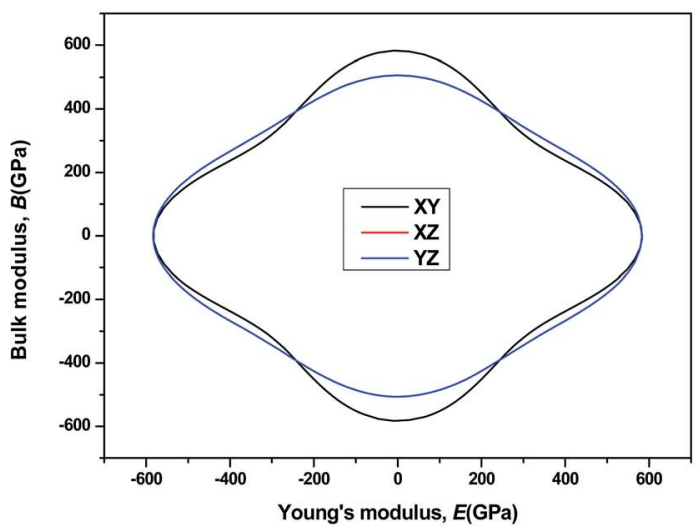

(d)

Fig. 7 Directional dependence of the Young's modulus for $\mathrm{Be}_{12} \mathrm{Ti}$ under $0 \mathrm{GPa}$ (a) and $40 \mathrm{GPa}$ (b). The corresponding projections in different planes ( $X Y$ plane, and $X Z$ plane, and $Y Z$ plane) of Young's modulus are also shown in (c) and (d).

\subsection{Thermodynamic properties}

We have used the quasi-harmonic approximation (QHA) to investigate the thermodynamic properties of tetragonal $\mathrm{Be}_{12} \mathrm{Ti}$ in the temperature range $0-1600 \mathrm{~K}$ and pressure range $0-$ $100 \mathrm{GPa}$, which has been successfully applied to several materials ${ }^{45-47}$ the detail calculation method are presented in ref. 46 and are not described here. To obtain the equilibrium volume and bulk modulus of $\mathrm{Be}_{12} \mathrm{Ti}$ at different temperature, we have calculated the Helmholtz free energy $F(V, T)$ dependence of the volume $V$, the equilibrium volume and the bulk modulus are obtained by above the formula (1) Birch-Murnaghan EOS. They are shown in Fig. 9 and 10, respectively. From Fig. 9, we can see that the free energy value decreases gradually and its change range in the same temperature interval (such as $200 \mathrm{~K}$ ) enhances as increase of temperature. If we connected the minimum values of free energy using one line we can obviously find that the equilibrium volume of $\mathrm{Be}_{12} \mathrm{Ti}$ expands with the increasing of temperature. Also, clearly, the bulk modulus decreases with the increase of temperature from $0 \mathrm{~K}$ to $1600 \mathrm{~K}$, and when the temperature is near $270 \mathrm{~K}$, the calculated result of bulk modulus are close to the experimental data.
The investigation on the heat capacity of crystal is an old topic of the condensed matter physics, ${ }^{48}$ because as one variation of the thermal properties, the heat capacity of a substance not only provides essential insight into its vibrational properties but is also mandatory for many applications. According to the laws of thermodynamics, there are two kinds of heat capacities in a material, namely heat capacity at constant volume $C_{\mathrm{v}}$ and heat capacity at constant pressure $C_{\mathrm{P}}$, the $C_{\mathrm{v}}$ and $C_{\mathrm{P}}$ can be calculated from ref. 46. Fig. 11 shows the variation of $C_{\mathrm{v}}$ and $C_{\mathrm{P}}$ with temperature at $0 \mathrm{GPa}$. One can easily see that $C_{\mathrm{v}}$ and $C_{\mathrm{P}}$ increases with the increase of temperature, and when temperature lower that $300 \mathrm{~K}$, they are almost equal, while the temperature is higher than $300 \mathrm{~K}, C_{\mathrm{p}}$ is always larger than $C_{\mathrm{v}}$, but the discrepancy between them becomes significant as the temperature enhances. Meanwhile, the constant volume $C_{\mathrm{v}}$ at various pressure is calculated and displayed in Fig. 12. It is obvious that the $C_{\mathrm{v}}$ at different pressure has a similar trend if compared with the $C_{\mathrm{v}}$ at $0 \mathrm{GPa}$. That is while $T<400 \mathrm{~K} C_{\mathrm{v}}$ increases rapidly with temperature at a given pressure and decreases with increasing pressure at a given temperature. Namely, the variation of $C_{\mathrm{v}}$ is dependent on both temperature and pressure at low temperature. At the same 


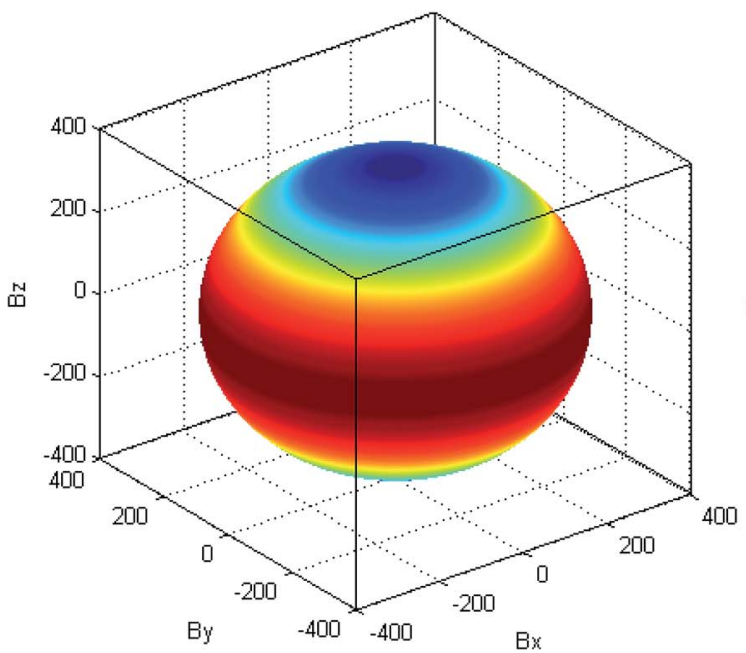

(a)

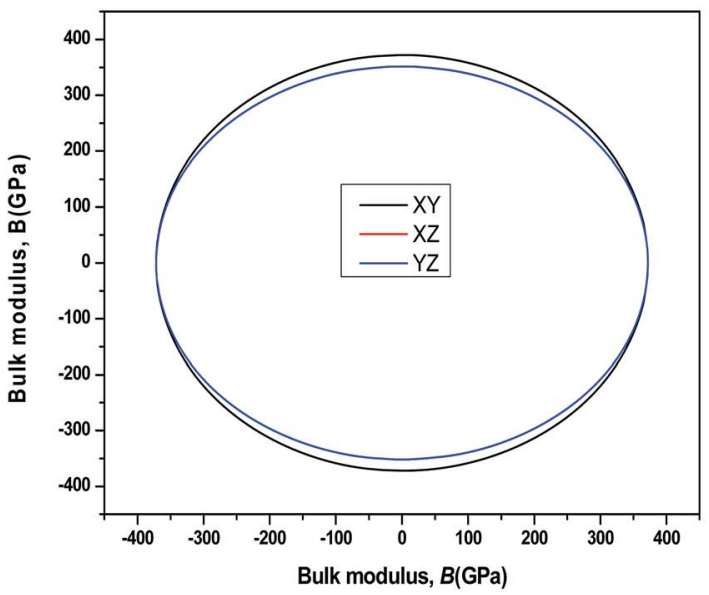

(c)

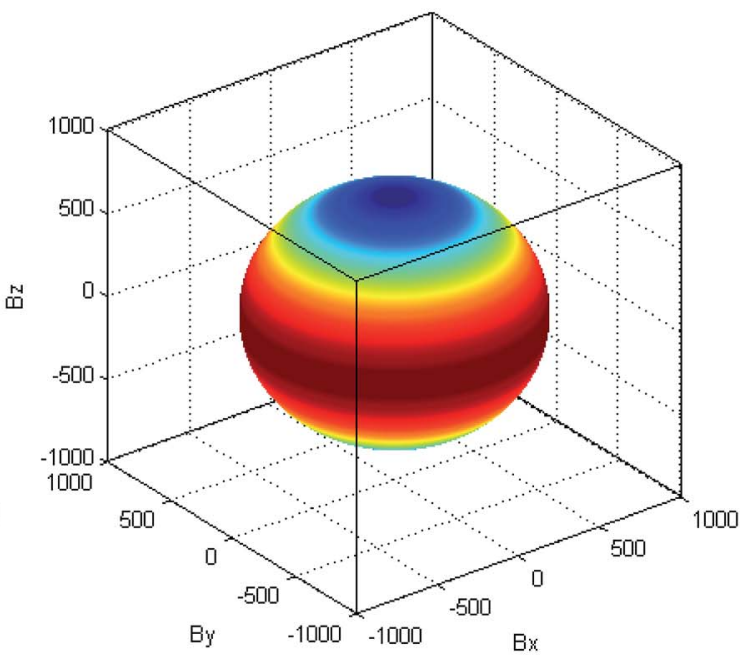

(b)

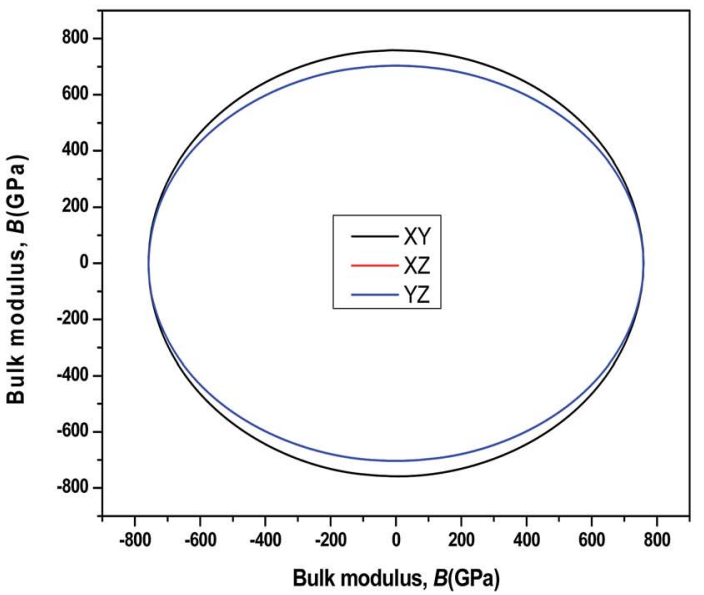

(d)

Fig. 8 Directional dependence of the Bulk modulus for $\mathrm{Be}_{12}$ Ti under $0 \mathrm{GPa}$ (a) and $40 \mathrm{GPa}$ (b). The corresponding projections in different crystal planes ( $X Y$ plane, and $X Z$ plane, and $Y Z$ plane) of Bulk modulus are also shown in (c) and (d).

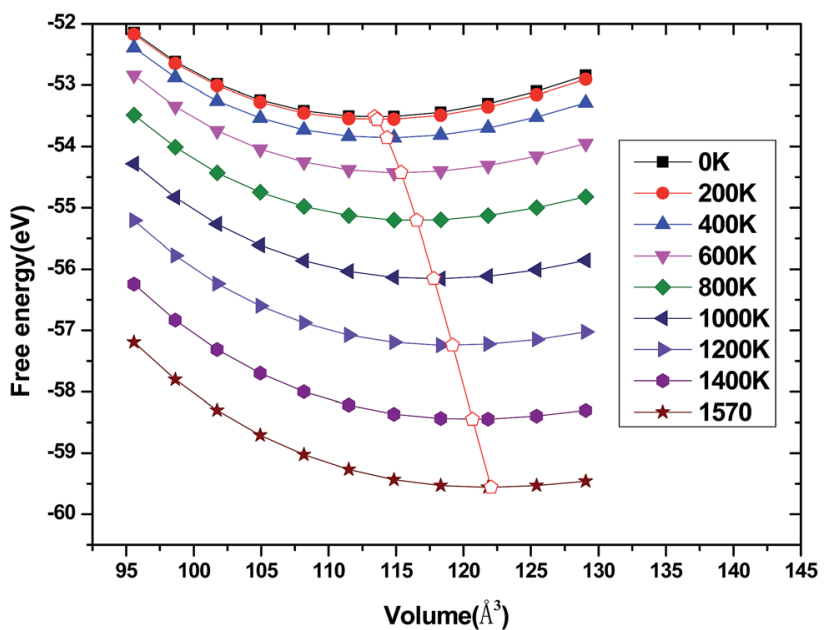

Fig. 9 Dependence of the Helmholtz free energy $F(V, T)$ on crystal volume at various temperatures and the locus of the minimum of the free energy for $\mathrm{Be}_{12} \mathrm{Ti}$.

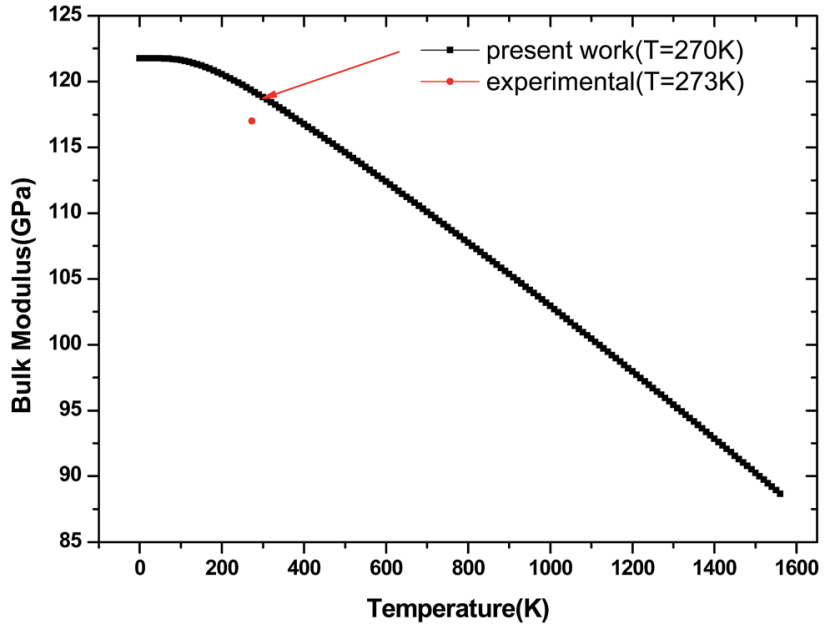

Fig. 10 Temperature dependence of the bulk modulus for $\mathrm{Be}_{12} \mathrm{Ti}$. 


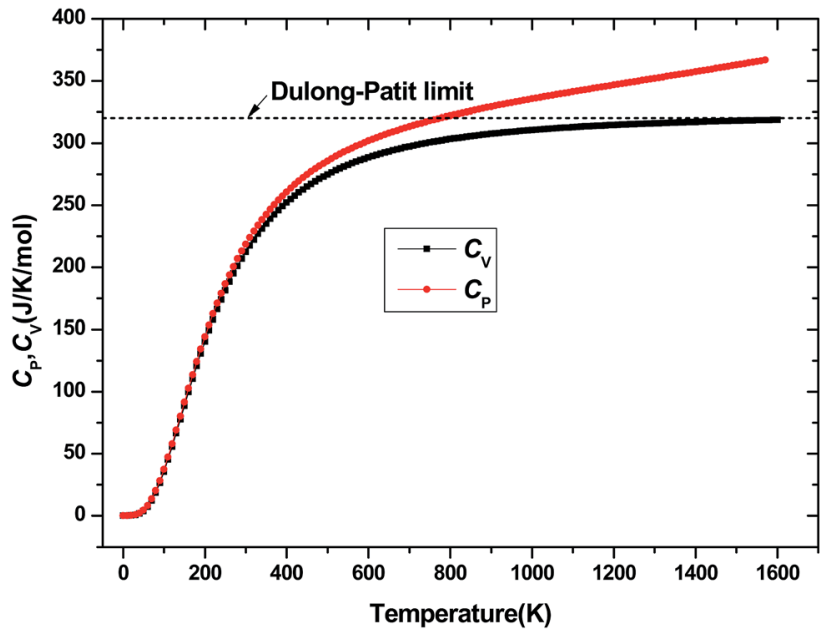

Fig. 11 Heat capacity of constant volume $C_{v}$ and constant pressure $C_{P}$ as a function of temperature at $0 \mathrm{GPa}$.

time at sufficiently low temperatures $(T<400 \mathrm{~K}), C_{\mathrm{v}}$ is approximately proportional to $T^{3} \cdot{ }^{49}$ However, the anharmonic effect on $C_{\mathrm{v}}$ is suppressed at higher temperature, the heat capacity $C_{\mathrm{v}}$ approaches to a constant Dulong-Petit limit $3 n N_{\mathrm{A}} K_{\mathrm{B}}(\approx 324.53 \mathrm{~J}$ $\left.\mathrm{mol}^{-1} \mathrm{~K}^{-1}\right),{ }^{33}$ where $n=13$ is number of atoms for $\mathrm{Be}_{12} \mathrm{Ti}$ unit cell, $N_{\mathrm{A}}$ is the Avogadro number and $K_{\mathrm{B}}$ is the Boltzmann's constant. At intermediate temperatures, the temperature dependence of $C_{\mathrm{v}}$ is governed by the details of vibrations of the atoms. It indicates that the atomic interaction occurs at low temperature in $\mathrm{Be}_{12} \mathrm{Ti}$ alloys. The effects of temperature on heat capacity are more significant than that of the pressure for $\mathrm{Be}_{12} \mathrm{Ti}$, and the $C_{\mathrm{v}}$ is more sensitive to the temperature than that of the pressure. Here, we also plotted the change of entropy with temperature at various pressure in Fig. 13 for $\mathrm{Be}_{12} \mathrm{Ti}$. It can be seen that the entropy increase quickly as increasing temperature and decreases with increasing of pressure. It's consistent with common sense, because the entropy of a crystal is generated by lattice vibrations and electronic excitation, and as the

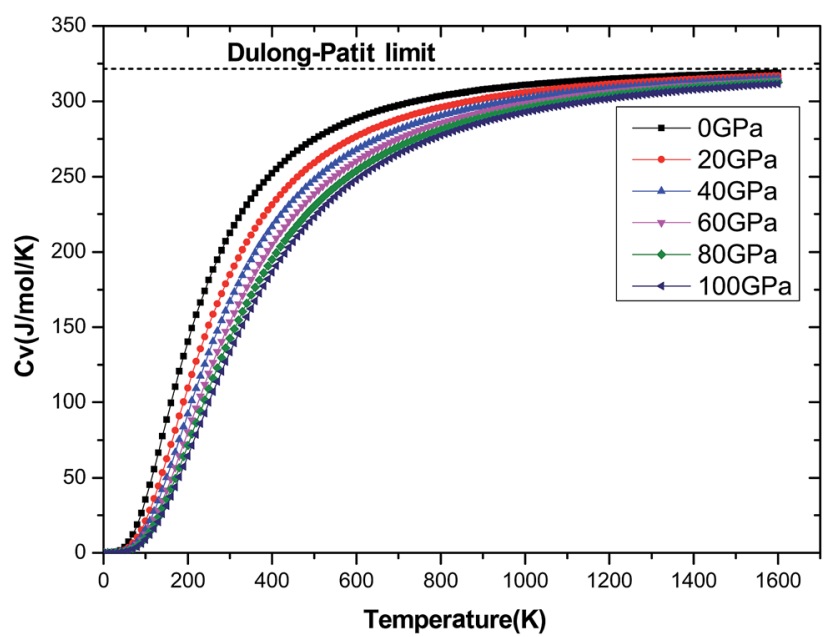

Fig. 12 Dependencies of the constant volume heat capacity $C_{v}$ on temperatures at high pressure.

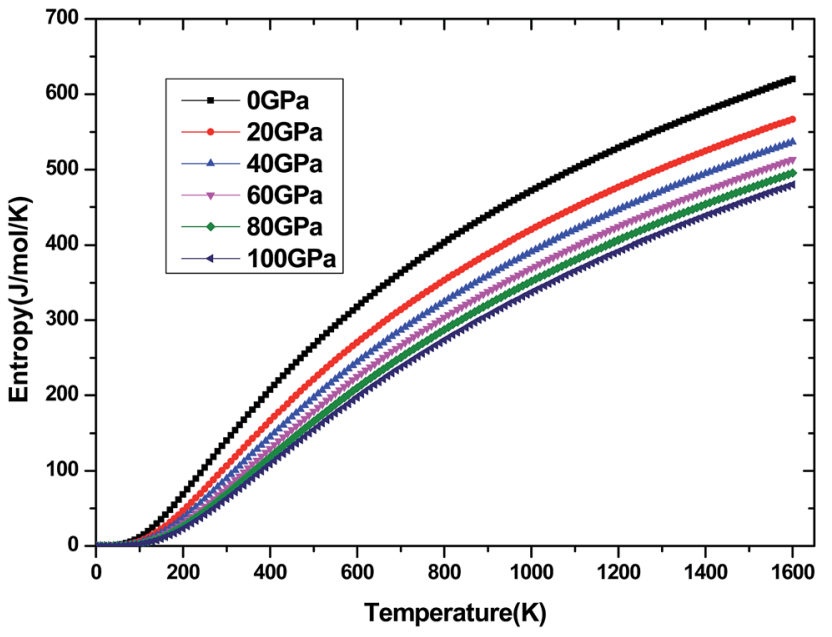

Fig. 13 Dependence of the entropy $S$ on temperature at high pressure.

temperature goes up, the lattice vibrations of the crystal increases and the electrons are more likely to be excited.

The dependence of the thermal expansion $\alpha$ with temperature $T$ and pressure $P$ is illustrated in Fig. 14. It is similar to the heat capacity, when the temperature $T$ is below $400 \mathrm{~K}$, $\alpha$ rises sharply with temperature, the propensity of increment becomes gently and gradually approaches to a liner increase at high temperature. For comparison, the experimental values of thermal expansion for tetragonal $\mathrm{Be}_{12} \mathrm{Ti}$ from ref. 50 are also presented in Fig. 14. The comparison demonstrates that our calculated results are in excellent agreement with the experimental data in the temperature range 300-800 K. This further indicates that the above calculation method and model for tetragonal $\mathrm{Be}_{12} \mathrm{Ti}$ are reliable and effective. Unfortunately, the experimental data are only in the range of 300-800 K, and more experimental values under high temperature are expected to be compared in the future.

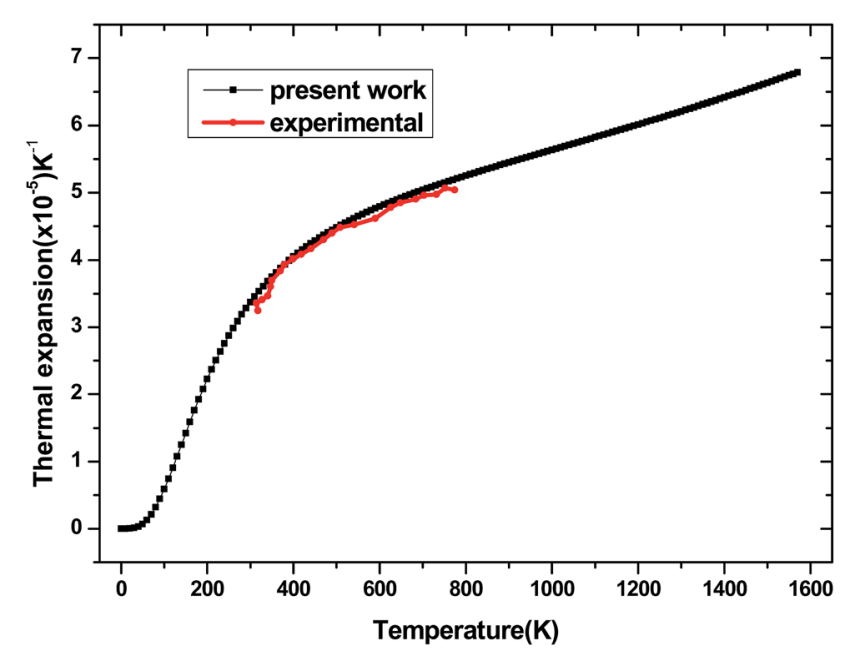

Fig. 14 The thermal expansion $\alpha$ of tetragonal $\mathrm{Be}_{12} \mathrm{Ti}$ as a function of temperature. 


\section{Conclusions}

In this work, the structure, elastic anisotropy and thermodynamic properties of tetragonal $\mathrm{Be}_{12} \mathrm{Ti}$ at different temperature and pressure are investigated using the pseudo-potential planewave method in combination with the quasi-harmonic approximation. The calculated equilibrium structure parameters and bulk modulus are good agreement with the available experimental data and other theoretical results. The calculated elastic constants $C_{i j}$ and interrelated quantities $G, E, \nu$ show that $\mathrm{Be}_{12} \mathrm{Ti}$ alloy possesses brittle nature at ambient conditions and $\mathrm{Be}_{12} \mathrm{Ti}$ exhibits no pronounced elastic anisotropy by the $3 \mathrm{D}$ surface of Young's modulus. The higher the temperature, cell compression for $\mathrm{Be}_{12} \mathrm{Ti}$ at a given pressure is more difficult. Phonon dispersions has been carried out, shows that the $\mathrm{Be}_{12} \mathrm{Ti}$ crystal is dynamic stability up to $100 \mathrm{GPa}$. Under the QHA method, the thermodynamic properties of $\mathrm{Be}_{12} \mathrm{Ti}$ are obtained successfully. The pressure and temperature dependence of heat capacity and thermal expansion are also predicted in the pressure ranges of 0-100 GPa and temperature range of 0-1600 K. Heat capacity $C_{\mathrm{v}}$ of different pressure is found to increase with temperature and close to the Dulong-Petit limit at high temperatures. Furthermore, thermal expansion $\alpha$ enhances with temperature but the variety tendency get slower at high temperature, and they are in excellent agreement with experimental data.

\section{Conflicts of interest}

There are no conflicts to declare.

\section{Acknowledgements}

The author would like to thanks Dr C. Cao and Dr Z. H. Yuan and J. M. Yan for his useful discussions about anisotropy elasticity. This project was supported by the National Magnetic Confinement Fusion Science Program of China under No. 2015 GB109005.

\section{References}

1 V. Chakin, M. Klimekov, R. Rolli, P. Kurinskiy, A. Moeslang and C. Dorn, J. Nucl. Mater., 2011, 417, 769.

2 K. Wada, K. Munakata, J. H. Kim, K. Yonehara, D. Wakai and M. Nakamichi, J. Nucl. Mater., 2013, 442, 5494.

3 T. Otsuka, T. Tanabe and K. Tokunaga, J. Nucl. Mater., 2013, 438, S1048-S1051.

4 Y. K. Vohra and P. T. Spencer, Phys. Rev. Lett., 2001, 86, 3068. 5 B. T. Wang, W. D. Li and P. Zhang, J. Nucl. Mater., 2012, 420, 501.

6 M. Grosse, G. Kuehne, M. Steinbrueck, E. Lehmann, J. Stuckert and P. Vontobel, J. Phys.: Condens. Matter, 2008, 20, 104263.

7 C. Li, C.-L. Feng, H. Y. Oderji, et al., Review of LIBS application in nuclear fusion technology, Front. Phys, 2016, 11(6), 114214.
8 W. Liu, B. Li, L. Wang, J. Zhang and Y. Zhao, Phys. Rev. B: Condens. Matter Mater. Phys., 2007, 76, 144107.

9 M. T. Perez-Prado and A. P. Zhilyaev, Phys. Rev. Lett., 2009, 102, 175504.

10 S. H. Zhang, Y. Zhu, X. Y. Zhang, S. L. Zhang, Q. Li and R. P. Liu, Comput. Mater. Sci., 2010, 50, 179.

11 S. H. Zhang, X. Y. Zhang, Y. Zhu, S. L. Zhang, Q. Li and R. P. Liu, Comput. Mater. Sci., 2012, 61, 42.

12 O. Rapp, J. Less-Common Met., 1970, 21, 27.

13 J. Z. Liu, A. van de Walle, G. Ghosh and M. Asta, Phys. Rev. B: Condens. Matter Mater. Phys., 2005, 72, 144109.

14 H. Kawamura, H. Takahashi, N. Yoshida, Y. Mishima and K. Ishida, J. Nucl. Mater., 2004, 329, 112.

15 V. Chakin, M. Klimenkov, R. Rolli and P. Kurinskiy, J. Nucl. Mater., 2011, 417, 769.

16 M. Uchida, E. Ishitsuka and H. Kawamura, J. Nucl. Mater., 2002, 307, 653.

17 P. Kurinskiy, A. Moeslang, V. chakin, M. Klimenkov, R. Rolli, S. Van Til and A. A. Goraieb, Fusion Eng. Des., 2013, 88, 2198. 18 S. M. Peng, J. Nucl. Mater., 2015, 464, 230.

19 X. K. Liu, W. Zhou, X. Liu and S. M. Peng, RSC Adv., 2015, 5, 59648.

20 X. L. Yuan, D. Q. Wei, X. R. Chen, Q. M. Zhang and Z. Z. Gong, J. Alloys Compd., 2011, 509, 769.

21 M. Mbarki and A. Rebey, J. Alloys Compd., 2012, 530, 36.

22 Z. W. Yang, D. M. Shi, B. Wen and R. Melnik, J. Alloys Compd., 2012, 524, 53.

23 G. Yao, Y. D. Liu, W. B. Zhang, X. Y. An and Y. Chen, J. Alloys Compd., 2013, 5081, 109.

24 G. Sai Gautam and K. C. Hari Kumar, J. Alloys Compd., 2014, 587, 380.

25 R. R. Hao, X. Y. Zhang and J. Q. Qin, RSC Adv., 2015, 5, 77399.

26 B. B. Zheng, M. G. Zhang and H. G. Luo, AIP Adv., 2015, 5, 037123.

27 Y. Wang, C.-M. Hao, H.-M. Huang, et al., Elastic, dynamical, and electronic properties of $\mathrm{LiHg}$ and Li3Hg: first-principles study, Front. Phys., 2018, 13(2), 137102.

28 G. Kresse and J. Furthmüller, Phys. Rev. B: Condens. Matter Mater. Phys., 1996, 54, 11169.

29 G. Kresse and D. Joubert, From ultrasoft pseudopotentials to the projector augmented-wave method, Phys. Rev. B: Condens. Matter Mater. Phys., 1999, 59, 1758.

30 P. E. Blöchl, Projector augmented-wave method, Phys. Rev. B: Condens. Matter Mater. Phys., 1994, 50, 17953.

31 F. Birch, J. Geophys. Res., B, 1978, 83, 1257.

32 S. I. Shang, G. Sheng, et al., Phys. Rev. B: Condens. Matter Mater. Phys., 2009, 80, 052102-052105.

33 R. Hill, Proc. Phys. Soc., London, Sect. A, 1952, 65, 349.

34 K. Liu, B. Dong, X. L. Zhou, S. M. Wang, Y. S. Zhao and J. Chang, J. Alloys Compd., 2015, 632, 830.

35 A. Zalkin, E. Sands, R. G. Bedford and O. H. Krikorian, Acta Crystallogr., 1961, 14, 63.

36 R. L. Fleischer and R. J. Zabala, Metall. Trans. A, 1989, 20, 1279.

37 J. P. Poirier, Introduction to the Physics of the Earth's Interior, Cambridge University Press, Oxford, 2000, vol. 39. 
38 C. V. Sinko and N. A. Smirnow, J. Phys.: Condens. Matter, 2002, 14, 6989.

39 W. C. Hu, Y. Liu, D. J. Li, X. Q. Zeng and C. S. Xu, Comput. Mater. Sci., 2014, 83, 27.

40 S. F. Pugh, Philos. Mag., 1954, 45, 823.

41 R. R. Hao, X. Y. Zhang, J. Q. Qin, J. L. Ning, S. H. Zhang, Z. Niu, M. Z. Ma and R. P. Liu, RSC Adv., 2015, 5, 77399.

42 X. Ji, Y. Yu, J. Y. Ji, J. P. Long, J. P. Chen and D. J. Liu, J. Alloys Compd., 2015, 623, 304.

43 S. I. Ranganathan and M. Ostoja-Starzewski, Phys. Rev. Lett., 2008, 101, 055504.

44 J. F. Nye, Physical Properties of Crystals, Clarendon Press, Oxford, 1985.
45 Y. Lu, D. F. Li, B. T. Wang, R. W. Li and P. Zhang, J. Nucl. Mater., 2011, 408, 136.

46 R. Wang, S. F. Wang and X. Z. Wu, Intermetallics, 2013, 43, 65.

47 X. L. Wen, Y. X. Liang, P. P. Bai, B. W. Luo, T. Fang, L. Yue, T. An, W. Y. Song and S. Q. Zheng, Physica B Condens. Matter., 2017, 525, 119.

48 T. Belaroussi, T. Benmessabih, F. Hamdache and B. Amrani, Physica B Condens. Matter., 2008, 403, 2649.

49 J. P. Poirier, Introduction to the Physics of the Earth's Interior, Cambridge University Press, Oxford. 2000, vol. 39.

50 M. L. Jackson, P. A. Burr and R. W. Grimes, Acta Crystallogr., Sect. A: Found. Adv., 2016, B72, 277-280. 